\title{
Common Generative Adversarial Network Types and Practical Applications *
}

\author{
Mehmet Ali Barışkan ${ }^{1}$, Zeynep Orman ${ }^{2}$ and Rüya Şamlı ${ }^{2 *}$ \\ ${ }^{I}$ Computer Engineering Dept./Faculty of Engineering \& Architecture, Istanbul Gelisim University, Turkey \\ ${ }^{2}$ Computer Engineering Dept./Faculty of Engineering, Istanbul University-Cerrahpasa, Turkey
}

(Conference Date: 5-7 March 2020)

(DOI: 10.31590/ejosat.araconf70)

ATIF/REFERENCE: Barışkan, M. A., Orman, Z. \& Şamlı, R. (2020). Common Generative Adversarial Network Types and Practical Applications. Avrupa Bilim ve Teknoloji Dergisi, (Special Issue), 585-590.

\section{Öz}

Bu çalışmada ele alınan Generatif Karşıılıklı Ağları (Generative Adversarial Networks - GAN), Kısıtlı Boltzmann Makineleri (Restricted Boltzmann Machines - RBM), Derin Boltzmann Makineleri (Deep Boltzmann Machines - DBM) ve Varyasyonel Otomatik Kodlayıcılar (ariational Autoencoders- VAE) gibi genel derin öğrenme algoritmalarının kısıtlamalarının üstesinden gelmek için geliştirilmiş birçok derin öğrenme yöntemi arasında yer almaktadır. GAN modelleri ve yapıları toplanan veri kümelerinden yeni benzersiz veriler oluşturabilir. Bu veri kümeleri kimi zaman binlerce veriden oluşabilir, veri kümelerinin içerisinde farklı türde veriler mevcut olabilir. Bu yöntemlerin varyasyonları, çoğunlukla görüntü restorasyonu ve görüntü oluşturma, sinyal işleme ve siber saldırıların tespiti gibi derin öğrenme uygulamaları için kullanılır. Literatürde pek çok farklı GAN modelleri mevcuttur. Bu çalışmada da, esas olarak En Küçük Kareler Oluşturucu Düşman Ağları (Least Squares Generative Adversarial Networks - LSGAN) ve Derin Konvolüsyonel Üretken Düşman Ağları (Deep Convolutional Generative Adversarial Networks - DCGAN) olarak adlandırılan iki GAN yöntemi üzerine odaklanılmışır. Bu farklı yöntemler, geleneksel GAN algoritmasının performansını iyileştirmek ve çeşitli problemlerin farklı gereksinimlerini karşılayarak çözmek için geliştirilmişlerdir. Bu çalışmada bu yöntemlerin mimarîleri, kullanılış biçimleri, özellikleri, sayısal tanımlamaları verilmiş ve birbirlerinden farkları açıklanmıştır. Bu çalışmada ayrıca her iki GAN yöntemi (LSGAN ve DCGAN) için de, toplanan verilerden yeni ve benzersiz veriler oluşturmak için kullanılan bu algoritmaların literatürdeki pratik uygulamaları da ele alınmıştır. LSGAN için literatürdeki 5 farklı çalışma, DCGAN için ise literatürdeki 2 farklı çalışma ele alınarak incelenmiştir. Son olarak, bu yöntemlerle elde edilen sonuçlar karşılaştırılmış ve hangi yöntem için hangi uygulamanın kullanılabileceği açıklanmıştır. Araştırmalardan görüldüğü üzere her iki yöntemin de uygulanabileceği problemler birbirinden farklılık göstermektedir.

Anahtar Kelimeler: Derin Öğrenme, GAN, LSGAN, DCGAN.

\section{Common Generative Adversarial Network Types and Practical Applications}

\begin{abstract}
Generative Adversarial Networks (GAN) which are analyzed in this study are among many deep learning methods which have been developed to overcome the restrictions of generic deep learning algorithms such as Restricted Boltzmann Machines (RBM), Deep Boltzmann Machines (DBM) and Variational Autoencoders (VAE). GAN models and structures can create new unique data from the collected data bases. These data bases can contain thousands of data and different types of data. The variations of these methods are mostly used for deep learning applications such as image restoration and creation, signal processing, and detection of cyber-attacks. In the literature, there are many different types of GANs. In this paper, it was focused on two GAN methods which are the Least
\end{abstract}

* This paper was presented at the International Conference on Access to Recent Advances in Engineering and Digitalization (ARACONF 2020). 
Squares Generative Adversarial Networks (LSGAN), and Deep Convolutional Generative Adversarial Networks (DCGAN). These methods have been developed to improve the performance of the traditional GAN algorithm and solve various problems by satisfying different requirements. In this study, the architectures, usage types, properties and numeric definitions about these two methods were given and also the differences between them were analyzed. After that, the practical applications of these algorithms in the literature which have been used for creating new and unique data from the collected data were also discussed in this paper. 5 literature studies for LSGAN and 2 literature studies for DCGAN were given. Finally, we have compared the obtained results of these methods and explain which method can be used for which type of application. As seen from the researches, the applications that these methods can be applied are different from each other.

Keywords: Deep Learning, GAN, LSGAN, DCGAN.

\section{Introduction}

Deep learning initiated a profound reform and has been applied to various real-world tasks such as image classification, object planning and segmentation. These tasks clearly fall within the scope of supervised learning. However, supervised learning processes can be unmanaged because of the productive models. Besided these deep productive models, some other models such as RBMs, DBMs and VAEs have been proposed. These models face the difficulty of intractable functions or the difficulty of intractable inferences, which in turn restricts the effectiveness of these models [1].

GANs are like drawing a portrait or creating a symphony. These tasks are known to be difficult when compared to other deep learning applications. It is much easier to define a painting by a painting or by people. However, it brings us closer to intelligence when computers are used. We have to hire a lot of artists to create animations in developing games. While creating the animations, there are some routine tasks. By implementing automation with GAN, we can focus on creative parties rather than repeating daily routine tasks [1].

As GAN takes data to the correct side of the decision boundry whatever it has the value of true or false, sometimes the real data can be more unrealistic than the created fake data. In the literature, it can be seen that there are many types of GAN algorithms. Also, these diversions of the GAN algorithms are necessary because of different application requirements. In this study, we tried to determine which GAN type must be preferred in different applications by considering the different types of GANs, and recent studies have been examined to shed light on the researchers working on this subject.

This study consists the following sections. In the second section, we described the most used GAN methods. In the third section, we examined the applications of LSGAN and in the fourth section, we examined the DCGAN applications and in the last section the conclusions were presented.

\section{Material and Method}

\subsection{GAN, LSGAN and DCGAN}

The learning process of GANs raises a discriminatory D and a generator G. The goal of G is to learn the distribution of pg at the beginning of $\mathrm{x}$. G starts from the pz (z) sampling of input variables $\mathrm{z}$ from the uniform or Gaussian distribution, then maps the input variables $z$ to the data variable $z \mathrm{G}(\mathrm{z} ; \theta \mathrm{g})$ on a differentiable network. $\mathrm{D}$, on the other hand, is a $\mathrm{D}$ (x; d) classifier that aims to recognize whether an image comes from the training data or G. Basic algorithm of GAN is shown as figure 1[1].



Fig. 1 GAN Algorithm 
The minimum target for GANs can be formulated as follows:

$\left.\min _{G} \max _{D} V_{G A N}(D, G)=\mathbb{E}_{x \sim p_{\text {data }(x)}}[\log D(x)]+\mathbb{E}_{z \sim p_{z(z)}}[\log 1-D(G(z)))\right]$

DCGAN combines a CNN using supervised learning with a GAN using an unsupervised learning algorithm [2].

Traditaional GANs, which define the discriminator as a classifier, adopt sigmoid cross-entropy loss function. When updating the generator, this loss function will cause loss degrees problem for samples that are on the right side of the decision limit but are still far from the actual data. To solve this problem, Mao et al. have recommended Least Squares Productive Competitive Networks (LSGAN). Assuming that they have used the A-b coding scheme for discriminatory, where a and $\mathrm{b}$ are labels for fake and real data respectively [3], then the objective functions of LSGANs can be defined as follows:

$\min _{D} V_{L S G A N}(D)=\frac{1}{2} \mathbb{E}_{x \sim p_{\text {data }}(x)}\left[(D(x)-b)^{2}\right]+\frac{1}{2} \mathbb{E}_{z \sim p_{z}(z)}\left[(D(G(z))-a)^{2}\right]$

$\min _{G} V_{L S G A N}(G)=\frac{1}{2} \mathbb{E}_{Z \sim p_{Z}(z)}\left[(D(G(z))-c)^{2}\right]$

\subsection{Applications of LSGAN}

In this section some papers about applications of LSGAN were given. The applications are: general application of LSGAN, sketch simplification based on conditional random field and LSGAN, application of improved LSGAN for rail crack detection by AE technique, determining the effectiveness of LSGAN and finally a new mimicking attack by LSGAN.

\subsubsection{General Application of LSGAN (2017)}

This paper was written by Mao et al. [3]. In this study, the authors presented LSGAN as a new model which they proposed. They used LSUN, CIFAR-10 and HWDB1.0 datasets. They got more detailed images on LSUN when compared to DCGAN. When they used CIFAR-10, they obtained 6,47 inception score out of 10 and when DCGAN was used, 6,22 out of 10 was obtained as the inception score. As human perspective, they used Amazon Mechanical Turk to make survey to ask people which images look more realistic and they got $56,4 \%$ of votes and $43,6 \%$ votes were taken by DCGAN.

\subsubsection{Sketch simplification based on conditional random field and LSGAN (2018)}

I $\mathrm{n}$ this study [4], the authors have used the sketches to make more realistic cartoons and game graphics. They have used different types of sketches such as Japanese Anime, Disney Style, Scene and architecture. The aim for the related article is to make easier to create graphics and cartoons. 


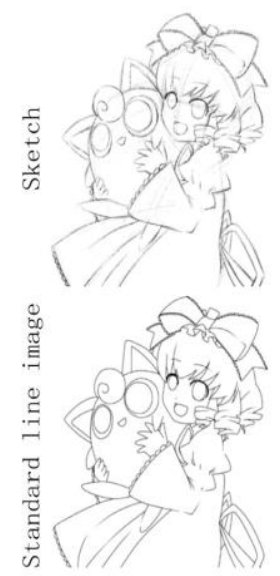

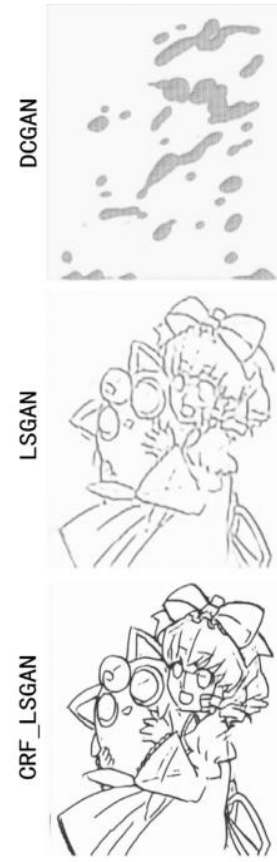

500 Iterations


1000 Iterations
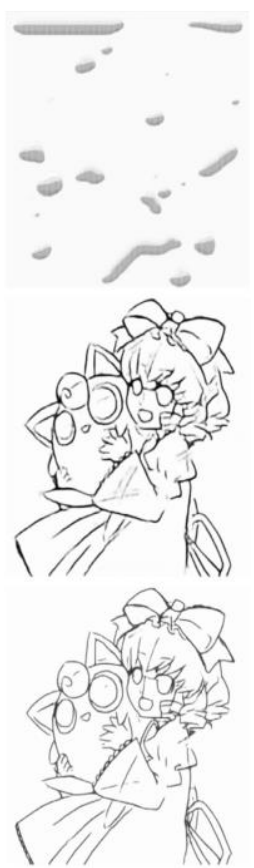

3000 Iterations

Fig. 2 Sample Outputs of three changing with iterations by Qianwen Lu,. 2018

In their experiments as given in Fig.2, they have compared the results of 3 algorithms: Deep Convolutional Generative Adversarial Networks (DCGAN), Least Squares Generative Adversarial Networks (LSGAN), and Least Squares Generative Adversarial Networks added the energy function of Conditional Random Field (CRF). Additionally, they have compared the proposed method and another method by combining LSGAN and fully connected CRF that was similar to the method proposed by Chen et al $[10]$.

\subsubsection{Application of improved LSGANs for rail crack detection by AE technique (2017)}

This study [5] was written by Wang et al. In this study the researchers have used sound files as the dataset. They have trained LSGAN using this dataset. The objective of this study is to find the deterioration of railroads much easier and to make the railroads safer. They have used the Gaussian mixture distribution dataset and they have obtained better results with the modified LSGAN then the Karman Algorithm.

\subsubsection{The Effectiveness of LSGANs (2018)}

This study [6] was written by Mao eta al. In this study, they have used least squares with discriminator \& generator. By using this approach, they obtained more clear pictures.

\subsubsection{A New Mimicking Attack by LSGAN (2017)}

This study [7] was written by Sun et al. In this study, they trained their LSGAN to understand the differences between Distributed Denial Service Attacks (DDOS) and Flash Crowds (FC) and created a simulation that could create artificial FC attacks. To test this method, they used the DDOS attack 2007 dataset for the DDOS attacks and the Word Cup 1998 dataset for the FC attacks. After testing, they found that even the most accurate defence mechanisms couldn't accurately (55\%) detect this type of attacks.

\subsection{Applications of DCGAN}

DCGAN consists one of the most popular and successful network designs for the GAN. It consists mainly of fold layers without maximum pooling or fully bounded layers. Evolutionary step and concave convolution [2] was shown in Fig 3. 

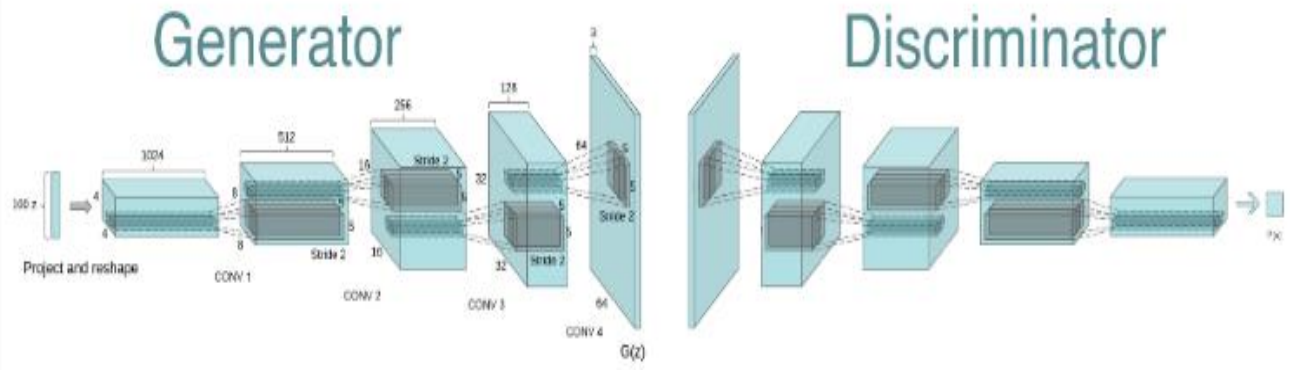

Fig. 3. DCGAN Generator Used For Lsun Scene Modeling (Alec Radford \& Luke, Metz 2016)

In this section some papers about applications of DCGAN were given. These papers are: DC-AL GAN: Pseudoprogression and true tumor progression of glioblastoma multiform image classification based on DCGAN and AlexNet and Semi-Supervised Encrypted Traffic Classification With DCGAN respectively.

\subsubsection{DC-AL GAN: Pseudoprogression and true tumor progression of glioblastoma multiform image classification based on DCGAN and AlexNet (2019)}

This paper [8] was written by Li et al. They have separated Pseudoprogression (PsP) which according the same article occurs in $20-30 \%$ of patients with glioblastoma multiforme and true tumor progression (TTP). They have used DCGAN and Alex-Net which was chosen as the discriminator, and it was used to identify the features needed for the final classification. AlexNet is an architecture based on CNNs that has proven to be successful in scene classification tasks. They have found that the accuracy of this approach is 0.920 for PSP, and 0.947 for TTP.

\subsubsection{Semi-Supervised Encrypted Traffic Classification With DCGAN (2019)}

This paper [9] was written by Iliyasu and Deng. They have used TensorFlow keras high-level API 2.0.0-alpha as the deep learning software framework to implement both the semi-supervised DCGAN model, and the baseline models. The hardware used was taken from Google Cloud Platform (GCP). They obtained results with $89 \%$ accuracy with $10 \%$ labelled data and $93 \%$ accuracy with $50 \%$ labelled data.

\section{Results}

A total of 7 articles have been found in the literature which have used Least Squares Generative Adversarial Networks (LSGAN), and Deep Convolutional Generative Adversarial Networks (DCGAN) as key terms in Web of Knowledge (WOS), Scopus, IEEE and Science Direct / Elsiever databases. 3 of these papers were published in 2017 whereas 4 of them were published in 2018 as shown in Fig. 4.

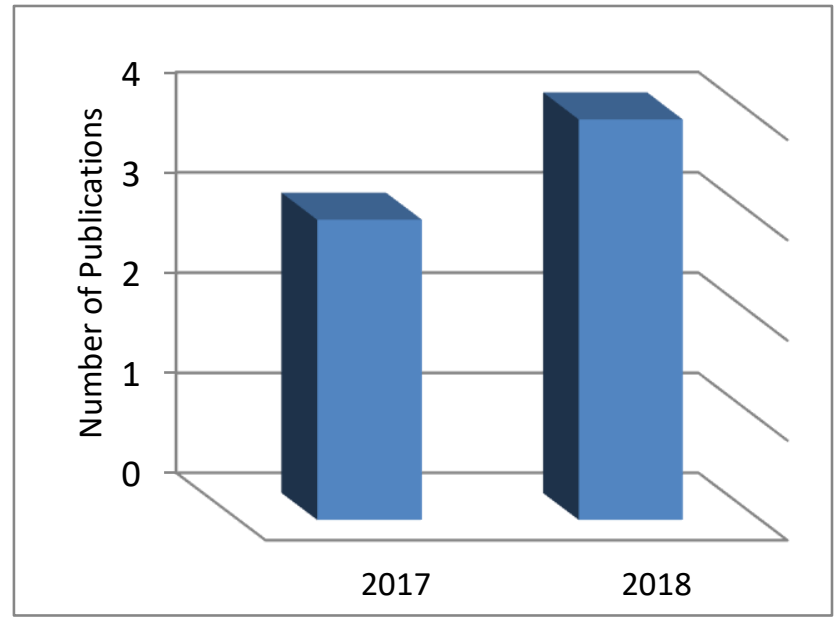

Fig. 4 Number articles by year 
While 3 of these articles including the one that has presented the GAN model for the first time are the conference papers, whereas 4 of them are journal papers as presented in Fig. 5.

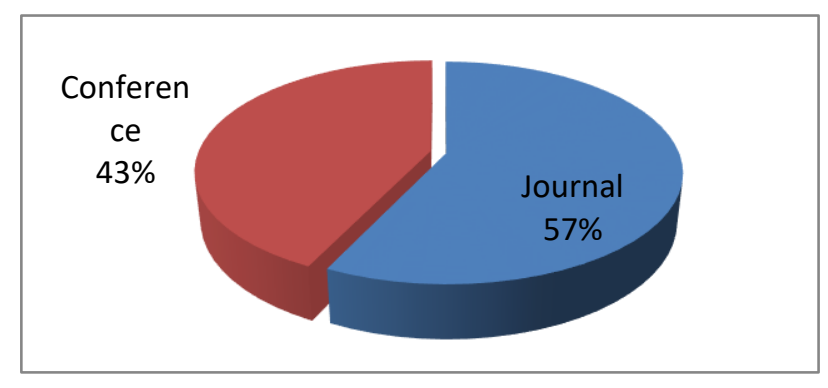

Fig. 5 Number of Articles by Publication Type

Because of the different requirements of the related problems, researchers and practitioners are going to need new GAN types. With the improvements of artificial intelligence and other practical areas of artificial neural networks and deep learning, it is obvious that there will be an increase in the types of the GAN algorithms. Therefore, there will be a variety of GAN algorithms that are being developed in the near future.

\section{Conclusion}

GAN is a model commonly used for estimating generative models by an adversarial process by simultaneously training two models. In this paper, we have examined the applications of the two most used GAN algorithms: LSGAN and DCGAN. We have found out that although LSGAN and DCGAN can be used in a similar manner, there are types of differences that make them suitable for different types of applications. While LSGAN is suitable for the scenarios such as duplication of train track sounds or cyber-attack simulations, in which we need perfect duplicates of the original data, DCGAN is suitable for creating new data that is not directly similar to main data such as medical researches and traffic management system simulations in which we need similar unique data to make comparisons. There are very few GAN algorithms developed in this area. As different applications will require different types of GAN algorithms, it is expected that studies on this subject will increase in the near future.

\section{References}

[1] Soumyadeep Kundu, Sayantan Paul, Suman Kumar Bera, Ajith Abraham, Ram Sarkar "Text-line extraction from handwritten document images using GAN” Expert Systems with Applications, Vol. 140, 112916, 2020.

[2] Soumith Chintala, Alec Radford, Luke Metz "Unsupervised Representation Learning With Deep Convolutional Generative Adversarial Networks"in Proc. ICLR 2016.

[3] Xudong Mao, Qing Li, Haoran Xie, Raymond Y.K. Lau, Zhen Wang, Stephen Paul Smolley, "Least Squares Generative Adversarial Networks" in Proc. ICCV 2017.

[4] Qianwen Lu, Qingchuan Tao, Yalin Zhao, Manxiao Liu "Sketch simplification based on conditional random field and least squares generative adversarial networks" Neurocomputing, Vol. 316, 178-189, 2017.

[5] Kangwei Wang, Xin Zhang, Qiushi Hao, Yan Wang, Yi Shen "Application Of Improved Least-Square Generative Adversarial Networks For Rail Crack Detection By Ae Technique” Neurocomputing, Vol. 332, 236-248, 2018.

[6] Xudong Mao, Qing Li, Haoran Xie, Raymond Y.K. Lau, Zhen Wang, Stephen Paul Smolley "On the Effectiveness of Least Squares Generative Adversarial Networks" IEEE Transactions On Pattern Analysis And Machine Intelligence, Vol. 41, 2019

[7] Degang Sun, Kun Yang, Zhixin Shi, Chao Chen, "A New Mimicking Attack By Lsgan”in Proc. IEEE 29th International Conference on Tools with Artificial Intelligence (ICTAI), 2017.

[8] Meiyu Li, Hailiang Tang, Michael D. Chan, Xiaobo Zhou, Xiaohua Qian "DC-AL GAN: Pseudoprogression and True Tumor Progression of Glioblastoma Multiform Image Classification Based on DCGAN and AlexNet" Cornell University arXiv: 1902.06085

[9] Auwal Sani Iliyasu, Huifang Deng, “A.Semi-Supervised Encrypted Traffic Classification With Deep Convolutional Generative Adversarial Networks"IEEE Access Vol.8, 118-126, 2019.

[10] Liang-Chieh Chen, George Papandreou, Iasonas Kokkinos, Kevin Murphy, Alan L. Yuille "Semantic image segmentation with deep convolutional nets and fully connected CRFs" in Proc. ICLR 2015. 